\title{
Rod and Screw Fixation for Cranio-Cervical Instability
}

\author{
Hedaya Hendam, Ahmed Taha* (D), Mohamed Youssef \\ Department of Neurosurgery, Al Azhar Faculty of Medicine, Damietta, Egypt \\ Email: hedayahendam@yahoo.com, *ahmdth231@gmail.com, youssef200877@yahoo.com
}

How to cite this paper: Hendam, H., Taha, A. and Youssef, M. (2020) Rod and Screw Fixation for Cranio-Cervical Instability. Open Journal of Modern Neurosurgery, 10, 19-26.

https://doi.org/10.4236/ojmn.2020.101003

Received: October 18, 2019

Accepted: November 9, 2019

Published: November 12, 2019

Copyright $\odot 2020$ by author(s) and Scientific Research Publishing Inc. This work is licensed under the Creative Commons Attribution International License (CC BY 4.0).

http://creativecommons.org/licenses/by/4.0/

\section{cc) (7) Open Access}

\begin{abstract}
Background Data: Atlanto-occipital dislocation is a rare and fatal condition. Pain, limitation of movements, and weakness, were the main complaints of patients with upper cervical lesions. Internal fixation and fusion was indicated in all patients of Atlanto occipital dislocation with deficits. CT scan is the imaging modality of choice for evaluation. Objective: To evaluate the efficacy and safety of rod and screw fixation in cranio-cervical instability. Study Design: Retrospective study reviewed all patient treated by rod and screw fixation, they were 12 patient operated for atlanto-occipital instability from April 2013 to April 2016 in Alazher University Hospital Damietta. Pre and postoperative data collection and analysis of the outcome were completed based on the Frankel classification and grading. Patients and Methods: We operated 12 patients, 10 with traumatic and 2 with pathologically diagnosed Atlanto occipital instability. From April 2013 to April 2016. All patients presented with neck pain, limitation of neck movement, and neurological deficits. The patients were investigated by standard plain $\mathrm{X}$ ray to the cervical spine, CT scan and MRI of the cervical spine pre-operatively, and they operated thorough posterior Cranio-cervical fixation. These patients followed post-operatively clinically for improving neural functions and radiologically for alignment, stability, fusion and efficacy of hard ware fixation. Results: The mean age of the studied cases was 42.1 years, trauma was the cause of instability in 10 patients, and 2 patients one with rheumatoid arthritis and one with neoplastic lesion. The mean follows up period is 14.7 months. We used screw rod system in posterior craniocervical fixation with iliac bone graft without operative or post-operative complications. All our patients were improved neurologically post-operatively and no hardware failure during the follow up period. Conclusion: Craniocervical instability was rare condition, with miss diagnosis and may be fatal condition. Posterior occipitocervical fixation when indicated can be done by various techniques. The screw rod system was the most upgraded used technique with immediate rigid fixation.
\end{abstract}


Surgery in this area was possible with confident results.

\section{Keywords}

Spine, Atlanto-Axial, Atlanto-Occipital, Cranio-Cervical Internal Fixation

\section{Introduction}

The anatomy of the occipitocervical junction involves very strong ligaments connecting the occiput to $\mathrm{C} 1$ and to $\mathrm{C} 2$. It therefore requires a very strong force to result in occipitocervical bony or ligamentous injuries [1] (Vinu 2016). Occipitocervical injuries must be suspected in high velocity injuries like RTAs and in the elderly with neck pain and stiffness following a fall [2] (Amar Saxen 2017). Occipitocervical dislocation injuries are relatively rare and account for approximately $1 \%$ of all injuries involving the cervical spine (Powers et al. 1979) [3]. However, these injuries are often severe and therefore tend to be fatal. In fact, they are thought to be underdiagnosed [4] (Byung et al. 2010). Conventional cervical spine plain films miss between 50\% - 93\% of occipital condyle fractures, which are also the most common cervical spine fracture missed by radiology residents. There is a need to have a high index of suspicion of such injuries in patients with dangerous mechanism of injury as elaborated by NICE (National Institute of Clinical Excellence) guidelines 2 (Amar Saxen 2017). There are a variety of ways to determine instability at the occipitoatlantal or atlantoaxial joints. Plain radiography is usually the first-line imaging modality. Findings related to occipitoatlantal translation, lateral atlas displacement, or atlantoaxial translation can raise suspicions of instability (Greene et al. 1997) [5]. Computed tomography (CT) improves definition of these articulations and can also detect rotational subluxation. Magnetic resonance imaging (MRI) can help determine ligamentous injury, especially of the transverse atlantal ligament (Dickman et al. 1996) [6].

The first preliminary report on successful use of a screw rod stabilizing system posteriorly applied to the occipitocervical junction was in 1996 by Jeanneret et al. [7]. In many studies, the clinical utility, safety, and effectiveness of hook-and-screw-rod and screw-and-rod instrumentation for posterior craniocervical fixation were evaluated. The best technique for occipitocervical fusion in case of an unstable craniocervical junction (CCJ) remains controversial since the desired rigid immediate stabilization demands increasing surgical complexity and risk (Menezes 1991) [8]. Pre-operative planning is essential and familiarity with different surgical techniques and implants allows the surgeon to choose the correct fixation method, giving the patients the best chance to heal [9] (R. Todd et al. 2015).

\section{Methods}

This retrospective study was carried out on 12 patients who underwent occipi- 
to-cervical fixation in Al-Azhar University new Damietta hospitals between April 2013 and April 2016. Their medical records and imaging studies were reviewed. All cases had preoperative craniocervical plain X-ray, CT and MRI examination. Preoperative traction was performed in two patients; one patient with trauma and the other with rheumatoid arthritis. All patients wore rigid neck collar postoperatively until fusion was achieved. All patients underwent occipitocervical fixation surgery with screw rood fixation systems and autologous bone grafts forfusion.

Surgical techniques

Preoperative

Intubation

Upon arrival to the operating room, patients were monitored with standard monitors. IV catheter was inserted in an upper extremity vein. Premedication with an atropine at least 20 minutes prior to the procedure was mandatory to prevent secretions from obscuring our view. After preoxygenation, anesthesia was induced with fentanyl $2 \mu \mathrm{g} / \mathrm{kg}$, propofol $2 \mathrm{mg} / \mathrm{kg}$, and atracurium $0.5 \mathrm{mg} / \mathrm{kg}$ and mask ventilation were done for 4 minutes. After the relaxant is given ample time to work, an assistant uses a cotton gauze to retract the tongue. The fiberoptic scope is then introduced into the oral cavity with a slight anterior curvature so the scope will follow the natural curvature of the oropharynx. The shaft of the scope is extended fully to maintain movements of the tip of the scope in the anterior-posterior plane. The shaft of the scope is braced against the 2 front teeth, which in most patients will maintain the scope in the midline. Once in the back of the throat (approx. $10-12 \mathrm{~cm}$ ), the uvula, tonsillar pillars laterally, the base of the tongue anteriorly and the tip of the epiglottis caudally should be visualized. If the tissues are collapsed at this point a gentle jaw lift will usually be helpful in revealing the tracheal inlet. Because the tracheal inlet is anterior to the esophagus, an anterior deflection of the tip is usually needed to advance the bronchoscope into the tracheal inlet. Just as the vocal cords are passed, the tip of the scope will usually visualize the anterior commissure of the vocal cords and anterior tracheal wall just caudal to the vocal cords. Once this has been achieved, and the vocal cords have been passed by the scope, the tip is deflected posteriorly and the scope advanced until the carina is visualized. The armored ET tube is then advanced into the trachea and the scope removed.

Then the patient was turned to the prone surgical position with the head rested on myefield head fixator maintaining neutral head position. The incisions were at midline, from external occipital protuberance to cervical area, after adequate exposure of sub occipital and posterior cervical areas. The methods used for occipitocervical fixation was screw-rod system in all cases. Cervical laminectomy was done in three cases depending on the clinical and radiological findings. We used lateral mass screws in most of the cases involving C3 and C4 and pedicular screws in $\mathrm{C} 2$, to keep accepted lordotic cranio-cervical junction. Due to early experience and little number of cases, we do not use C1 lateral mass 
screws in these cases. Finally, the autologous bone grafts harvested from posterior iliac crest were put between $\mathrm{C} 1$ and $\mathrm{C} 2$ or posterolaterally. The Frankel grade (Table 1) was used to assess neurological state preoperatively and postoperatively. Fusion was assessed by plain cervical X-ray films and CT scan. Fusion was defined as successful if 2 criteria were met: 1) there was no relative movement between the fused motion segments in dynamic views of cervical $\mathrm{X}$-ray; 2) the bone grafts became a uniform bone mass in imaging studies. The mean follow-up period was 14.7 months (range, 4 - 24 months).

\section{Results}

There were 9 males and 3 females, and their ages ranged from 16 to 63 years with mean age of 42.1 years. These series involved 10 patients with traumatic pathology (4 road traffic accident, 4 falls from height and 2 diving injury), one neoplastic lesion and one rheumatoid arthritis. These patients were followed mainly for 12.5 months (ranged 7 - 16 months). The clinical presentation, all patients had presented with neck pain before surgery, 8 patients with myelopathy, and 8 patients had presented with a neurological deficit either motor or sensory or both. All patients were improved post-operatively and reach to Frankel grade E from grade $\mathrm{C}$ and $\mathrm{D}$ pre-operatively, without neurological deficits (Frankel classification (Frankel, H.L. et al., 1969) [10] of these 12 patients are shown in Table 1).

As regard to the radiological finding of these cases: one patient had destructive neoplastic lesion in C2 body and left pedicle with evident cord compression, proved post-operatively plasmacytoma. One patient diagnosed pre-operatively as rheumatoid arthritis presented by well-formed pannus and ventral cord compression. The remaining 10 traumatic patients, radiologically diagnosed as 7 cases with Type 11 odontoid fracture (Figure 1) and 3 cases burst fracture C1 (Figure 2). These patients operated through posterior craniocervical fixation using screws-rod systems. Post-operatively all cases were followed for stability of the hard ware fixation system and efficacy of bone fusion in regular visit. There was no hard ware failure of the used screws rood system with accepted bony fusion in 10 traumatic cases, poor fusion in rheumatoid arthritis case and no observed fusion in the neoplastic case during the short period of follow up. There were no intra-operative or post-operative complications, no significant operative blood loss (less than $200 \mathrm{cc}$ ) and the operative time was 100 minutes at the start

Table 1. Frankel classification of 12 patients with occipitocervical instability.

\begin{tabular}{ccc}
\hline Frankel grade No. of cases & Preop. & Postop. \\
\hline A = complete paralysis & - & - \\
B = sensory function only below the injury level & - & - \\
C = incomplete motor function below injury level & 7 & - \\
D = fair to good motor function below injury level & 4 & 12 \\
E = normal function & 1 & - \\
\hline
\end{tabular}




\section{Case presentation:}

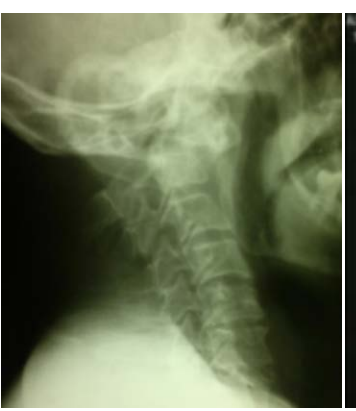

(a)

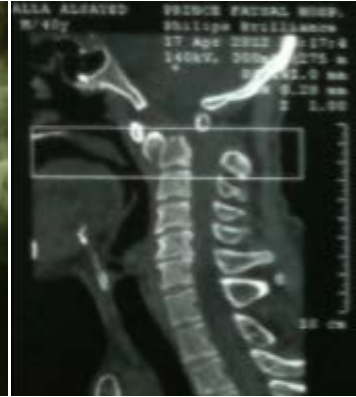

(b)

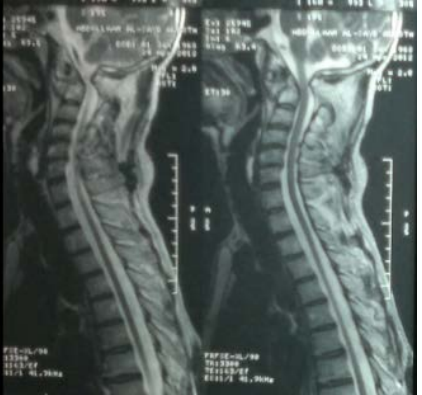

(c)

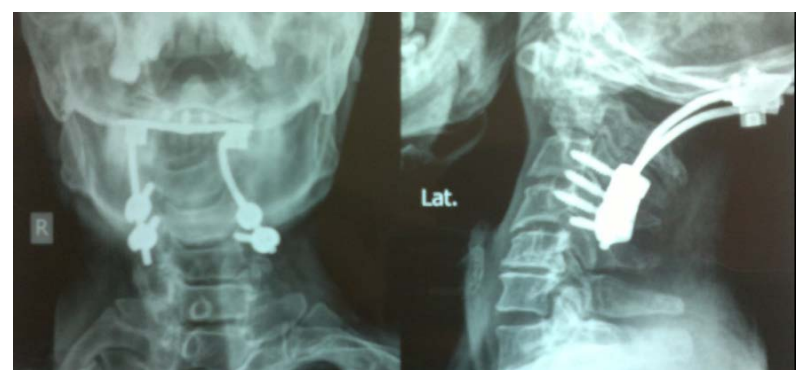

(d)

Figure 1. Male patient with (a) plain X ray cervical spine lateral view showing type 11 odontoid fracture, (b) saaggital reconstruction CT scan with anterior displacement of the fractured tip, (c) saggital MRI with upper cervical cord compression and (d) post-operative plain X ray AP and Lateral views after posterior occipito-cervical fixation involving the occiput and C3 and C4.

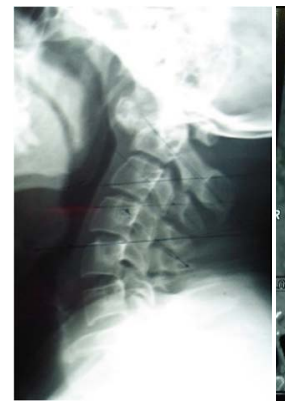

(a)

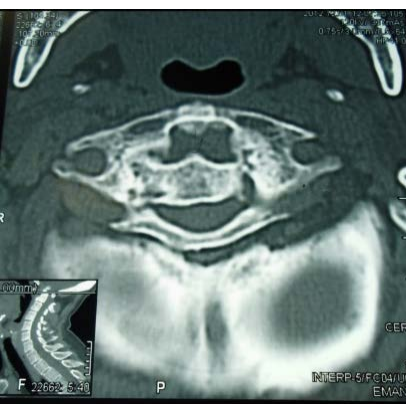

(b)

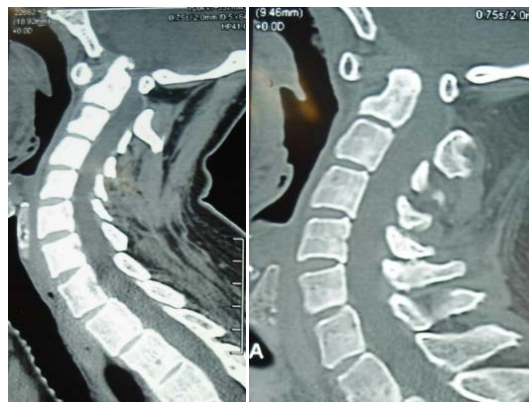

(c)

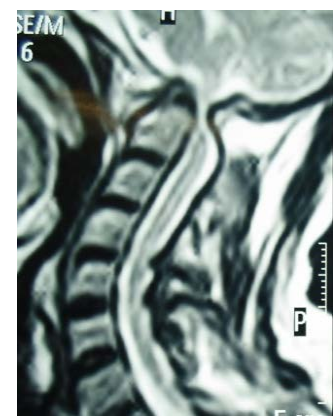

(d)

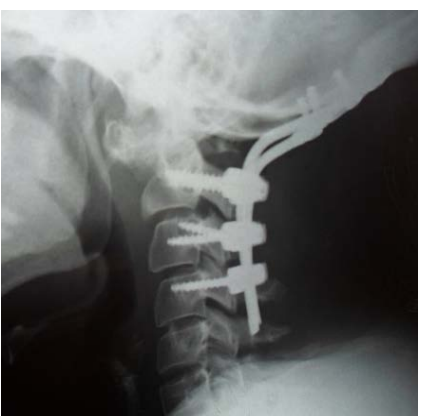

(e)

Figure 2. Male patient with (a) plain X ray of cervical spine lateral view with anterior and upward displacement of the dens and burst fracture of $\mathrm{cl}$, (b) axial CT scan cut opposite C1 with narrowed anteroposterior diameter, (c) saggital reconstruction CT scan with evident upward migration and posterior displacement of the dens, (d) MRI saggital view with marked upper cervical spinal cord compression and (e) post operative plain X ray of occipito-cervical fixation BY screw rod system included the occiput and C2 $\mathrm{C} 3$ and $\mathrm{C} 4$ with laminectomy $\mathrm{C} 1$ and foramen magnum decompression. 
of this surgery with reasonable short time (75 minutes) with more surgical experience.

\section{Discussion}

The atlanto-axial complex is more easily destabilized in certain pathological conditions. Significant atlanto-axial instability is a potentially serious progressive condition that, if untreated, may result in local pain, myelopathy or ultimately death (Hiroshi-Kuroki 2014) [11]. The success of cranio-cervical junction surgery depends up on adequate reduction, decompression of craniovertebral junction followed by immediate fixation of atlanto-axial joint with bone grafting and compression for solid bony fusion (Vinu et al. 2016) [1]. Occipitocervical fixation is indicated for OC instability or atlantoaxial instability where the patient is not candidate for the atlantoaxial arthrodesis [9] [12] (Ihab et al. 2011 and R. Todd et al. 2015).

The screw-based construct provides immediate postoperative stability, so postoperative rigid external fixation is not needed in most cases, which makes patients more comfortable. This construct provides more rigidity biomechanically than the wire-rod construct, and can be applied to patients whose posterior elements are defective or need to be removed for decompression. The disadvantages include potential vertebral artery and cervical root injury due to cervical screw purchase. In contrast, the screw-rod system is more rigid biomechanically [13] (Currier et al., 2003). The rod is contoured only in the sagittal plane, and the cervical screw can be inserted from its ideal entry point, resulting in optimal screw purchase with less incidence of neurovascular tissue injury. The rod is secured in an occipital plate fixed in the sub occipital midline ridge, which provides the strongest pullout strength for occipital fixation. In our experience, the screw-rod device offers some advantages, including strong occipital screw purchase, ideal cervical screw entry, easy contour of the rod to fit the occipitocervical curvature, and allowing cervical decompressive procedures [14] (Yu-Hone et al., 2009). As regard to our patient's number (12) was small like other series [4] [11] [14] [15] (Yu et al. 2009, Byung et al. 2010, George et al. 2011, and Hiroshi et al. 2014) and males were dominant in all series. The main age (41.2 years) of our patients was younger than in other series. The clinical presentation of our patients was of no difference of other series due to rarity of the cases. We used Frankle grading scale in clinical evaluation in agree with others as Ihab et al. 2011, but another authors use Nurick scale [14] (Yu et al. 2009), or Japanias orthopedic association score [4] (Byung et al. 2010). The indications for posterior craniocervical fixation were the same with all authors with different concepts about the used construct, with priority to the screw rod systems for fixation. In our series there were no intra-operative, post-operative complications nor surgery related deaths. This agreed of George et al. 2011 series, and with both series of Yu et al. 2009, and Vinu et al. 2016, except for single case post-operative infection in each series. Hirch et al. 2014, also recorded one case of failed fusion 
related to post-operative infection. Byung et al. 2010, reported one case of vertebral artery injury during the operation, and Ihab et al. 2011, reported 3 cases with post-operative transient neurological deterioration, two cases had superficial wound infection and CSF leak occurred in one case. Byung et al. 2010 also reported one patient died 2 months after surgery because pneumonia and sepsis. Bony fusion along short time of follow up (about fourteen months) was achieved in 10 patients who were similar or nearly equal to other series.

\section{Conclusion}

Craniocervical instability was rare condition, with miss diagnosis and may be fatal condition. Posterior occipitocervical fixation when indicated can be done by various techniques. The screw rod system was the most upgraded used technique with immediate rigid fixation. The bone fusion was essential in these techniques to save grad against delayed hardware failure. Pre-operative anatomical orientation by the craniocervical junction and screws occipital, lateral mass or pedicular entry points, with highly indicated patients for craniocervical fixation was the main points for successful surgery. Surgery in this area was possible with confident results.

\section{Conflicts of Interest}

The authors whose names are listed immediately below certify that they have no affiliations with or involvement in any organization or entity with any financial interest or non-financial interest in the subject matter or materials discussed in this manuscript.

\section{References}

[1] Gopal, V.V. (2016) Atlantoaxial Fixation-Anterior or Posterior Approach, Critical Review. The Journal of Spinal Surgery, 3, 51-54. https://doi.org/10.5005/jp-journals-10039-1087

[2] Saxena, A. (2017) Cranio-Cervical Trauma Epidemiology, Classification, Diagnosis and Management. Journal of Spine \& Neurosurgery, 6, Article ID: 1000284. https://doi.org/10.4172/2325-9701.1000284

[3] Powers, B., Miller, M.D. and Kramer, R.S. (1979) Traumatic Anterior Atlanto-Occipital Dislocation. Neurosurgery, 4, 12-17. https://doi.org/10.1227/00006123-197901000-00004

[4] Byung, S.K., Do, S.Y., Phil, W.H., Kyoung, S.C. and Sang, B.L. (2010) Atlantoaxial Transpedicular Screw Fixation for Management of Traumatic Upper Cervical Spine Instability. Journal of Korean Neurotraumatology Society, 6, 143-149. https://doi.org/10.13004/jknts.2010.6.2.143

[5] Greene, K.A., Dickman, C.A. and Marciano, F.F. (1997) Acute Axis Fractures: Analysis of Management and Outcome in 340 Consecutive Cases. Spine, 22, 1843-1852. https://doi.org/10.1097/00007632-199708150-00009

[6] Dickman, C.A., Greene, K.A. and Sonntag, V.K. (1996) Injuries Involving the Transverse Atlantal Ligament: Classification and Treatment Guidelines Based upon Experience with 39 Injuries. Neurosurgery, 38, 44-50. 
https://doi.org/10.1097/00006123-199601000-00012

[7] Jeanneret, B. (1996) Posterior Rod System of the Cervical Spine: A New Implant Allowing Optimal Screw Insertion. European Spine Journal, 5, 350-356.

https://doi.org/10.1007/BF00304352

[8] Menezes, A.H. (1991) Complications of Surgery at the Craniocervical Junction-Avoidance and Management. Pediatric Neurosurgery, 17, 254-266. https://doi.org/10.1159/000120607

[9] Todd Allen, R., Decker, R., Hong, J.T. and Sasso, R. (2015) Complications of Occipitocervical Fixation. Seminars in Spine Surgery, 21, 167-176.

https://doi.org/10.1053/j.semss.2009.05.006

[10] Frankel, H.L., Hancock, D.O., Hyslop, G., et al. (1969) The Value of Postural Reduction in the Initial Management of Closed Injuries of the Spine with Paraplegia and Tetraplegia. Spinal Cord, 7, 179-192. https://doi.org/10.1038/sc.1969.30

[11] Kuroki, H. (2014) Management of Atlantoaxial Instability-Surgical Strategy Based on Biomechanical View Point. Journal of Neurosurgery Spine, 2, 1008.

[12] Ihab, Z. and Wael, F. (2011) Occipitocervical Fixation in Management of Craniocervical Instabilities. Alexandria Journal of Medicine, 47, 185-192. https://doi.org/10.1016/j.ajme.2011.07.007

[13] Currier, B.L., Papagelopoulos, P.J., Neale, P.G., Andreshak, J.L., Hokari, Y., Berglund, L.J. and Larson, D.R. (2003) Biomechanical Evaluation of New Posterior Occipitocervical Instrumentation System. Clinical Orthopaedics and Related Research, 411, 103-115. https://doi.org/10.1097/01.blo.0000068760.86536.54

[14] Hsu, Y.-H., Liang, M.-L., Yen, Y.-S., Cheng, H., Huang, C.-I. and Huang, W.-C. (2009) Use of Screw-Rod System in Occipitocervical Fixation. Journal of the Chinese Medical Association, 72, 20-28. https://doi.org/10.1016/S1726-4901(09)70015-2

[15] George, S., Stamations, A., Dimitrious, S., Konstantinos, K., George, T. and Paclos, K. (2011) Posterior Instrumentations for Occipitocervical Fusion. The Open Orthopedics Journal, 5, 209-218. https://doi.org/10.2174/1874325001105010209 